\title{
El papel de las TIC en las administraciones públicas
}

\author{
Sr. Jose Manuel García Duarte \\ Director General de Tecnologías de la Información \\ y las Comunicaciones (DGTIC)
}

\section{Resumen}

La Dirección General de Tecnologías de la Información y las Comunicaciones (DGTIC) es el órgano de la Generalitat que asume las competencias, para la propia Administración de la Generalitat y sus organismos autónomos, en materia de modernización de la Administración, seguridad de la información, planificación, coordinación, autorización y control de las tecnologías de la información, las telecomunicaciones corporativas y la administración electrónica de la Generalitat.

La DGTIC es responsable de garantizar el derecho de acceso de los y las valencianas a los servicios de Tecnologías de la Información y la Comunicación (TIC) y de promover el desarrollo del sector tecnológico valenciano, por lo que impulsa modelos de colaboración entre universidades, institutos tecnológicos, empresas y Administraciones Públicas para el desarrollo de la sociedad digital.

En los últimos años la DGTIC ha hecho un esfuerzo redoblado por lograr esos objetivos. En primer lugar y hasta 2019, digitalizando la administración valenciana a un ritmo desconocido hasta ese momento. Y a continuación, llegado 2020, dando soporte tecnológico a los procesos de la administración y a las personas funcionarias en el desempeño de su trabajo durante la crisis sin precedentes que ha supuesto la pandemia de la COVID-19.

A estos dos impulsos sucesivos va a seguir un tercero que se extenderá entre 2021 y 2025: la recuperación de la crisis mediante la administración efectiva de los fondos derivados de los mecanismos de resiliencia y recuperación y la implementación del plan estratégico de transformación digital GEN Digital 2025.

\section{Transformación digital de la Generalitat desde 2015 hasta 2019}

La Generalitat, a través de la DGTIC, definió entre 2015 y 2016 un conjunto de planes en el ámbito de actuación de las TIC y la Administración electrónica que finalizaron en 2019.

El Plan de Transformación Digital de la Generalitat que, a su vez, incluía un Plan de Administración Electrónica, ha servido para dar el impulso necesario a la transformación de la Administración valenciana y desarrollar actuaciones que han permitido que la Generalitat cuente con numerosos casos de éxito. 
La Generalitat ha puesto el foco en priorizar las soluciones dirigidas a la ciudadanía y ha realizado un esfuerzo importante en aspectos de usabilidad y ergonomía de las herramientas con el fin de reducir la brecha digital que tanto preocupa, así como en dotar a los funcionarios de las herramientas básicas.

Desde la puesta en marcha del Plan de Administración Electrónica, el uso de la tramitación telemática ha aumentado considerablemente. En 2015, menos del 15\% de los trámites en la Generalitat eran plenamente electrónicos.

Este porcentaje ha ido en aumento constante hasta que, en 2017, se logró que la práctica totalidad de los trámites pudiese realizarse on-line, gracias a que se habilitaron los trámites generales para los casos en los que no existiese todavía un trámite específico en la sede electrónica.

Así, con la implementación del plan, entre 2016 y 2019 la tramitación electrónica ha crecido más del $80 \%$. A esto ha contribuido la plena implantación de la firma electrónica en la Generalitat para facilitar la certificación y verificación en la sede de documentos por parte de ciudadanos, empresas y organismos, incluida la propia Administración. Su uso ha pasado de una media de 400 firmas mensuales en 2016 a las más de 700.000 que se produjeron al mes en 2019.

Además, con la integración de la Generalitat en Cl@ve se ha facilitado a la sociedad valenciana la firma electrónica, también, desde dispositivos móviles y el uso de los servicios públicos digitales.

Su uso está muy extendido entre la ciudadanía y, solo en 2019 , se contabilizó un total de 5,4 millones de autenticaciones, posicionando a la Comunitat Valenciana como la comunidad autónoma en la que se realizó un mayor uso de la pasarela de Cl@ve.

En este sentido, el uso de la carpeta ciudadana, el área de usuarios de la ciudadanía que pone a disposición la Generalitat, casi se ha decuplicado (ha aumentado un 836\%) desde 2016, con más de 832.000 accesos anuales de ciudadanos y ciudadanas en 2019.

Entre 2017 y 2019, los pagos on-line crecieron un 150\%. Las notificaciones electrónicas han pasado de las 17.000 anuales de 2016 a las más de 375.000 de 2019. Además, la Comunitat es una de las tres únicas comunidades junto a Andalucía y Cataluña, que han integrado su plataforma propia de notificaciones en el Punto de Acceso General del Estado para poder consultar las notificaciones de la Generalitat desde la plataforma estatal.

La presentación electrónica de solicitudes con documentación (apuntes registrales) se ha multiplicado por 10 desde 2016 y en 2019 ha supuesto que cerca de un millón de personas se ahorrasen los desplazamientos a la Administración para presentar sus solicitudes.

Asimismo, la implantación del sistema de digitalización certificada en la Generalitat, para la emisión de copias auténticas, con plena cobertura legal, de los documentos que presentan los ciudadanos y ciudadanas en los registros de entrada, generó la digitalización con copia auténtica de más de 35.000 documentos en 2019, 
minimizando los costes de la manipulación y el tránsito del papel entre la ciudadanía y la Administración.

Además, el uso de los servicios de verificación publicados en la Plataforma Autonómica de Interoperabilidad (PAI) se ha multiplicado por 7 , ha crecido un $645 \%$.

Así, en 2017, el Consell aprobó el Reglamento de Administración Electrónica de la Comunitat Valenciana para adaptar la normativa de la Generalitat a la exigencia del nuevo paradigma digital en el que las relaciones con la Administración deben estar siempre apoyadas en los medios electrónicos.

Este mismo año, se ha publicado la nueva Política de Gestión de Documentos Electrónicos de la Generalitat (PGDE), que permite el tratamiento unificado de toda la información con la que trabajan los distintos departamentos de la Generalitat. Además, permite a la ciudadanía acceder fácilmente a sus expedientes, proteger sus derechos e intereses y mejorar la labor de la Administración evaluando su impacto.

Este año también, se presentó las bases de la Ley Valenciana de Desarrollo de la Sociedad Digital (LVDSD), que servirá de marco regulatorio para coordinar definitivamente el impulso a la transformación digital de la sociedad valenciana y que ahora es competencia de la Conselleria de Innovación y Sociedad Digital.

\section{Ciberseguridad}

En materia de ciberseguridad, la Generalitat ha mejorado y ampliado las capacidades y funciones del Centro de Seguridad TIC de la Comunitat Valenciana (CSIRT-CV) a través de un nuevo contrato que ha supuesto multiplicar por 3 la inversión en ciberseguridad (incremento del $195 \%$ ).

Con ello, se han aportado nuevas y mejores tecnologías y más recursos especializados para la mejora de la detección y respuesta ante las ciberamenazas, acordes al nuevo panorama global de la seguridad informática.

También desde CSIRT-CV, con el objeto de mejorar la cultura de la seguridad de toda la sociedad valenciana, se ha puesto en marcha el Plan Valenciano de Capacitación en Ciberseguridad.

Las primeras acciones para la ciudadanía han ido dirigidas a colectivos vulnerables, como las mujeres víctimas de violencia de género y los jóvenes y adolescentes. En concreto, el Plan de Acción para Centros Educativos ya ha permitido impartir cursos prácticos en 123 centros escolares, formando a más de 14.000 personas entre alumnos, padres y profesores.

\section{Redes y comunicaciones}

Se ha puesto en marcha el Plan de Banda Ancha de Alta Velocidad de la Generalitat y en este marco se ha publicado el mapa de banda ancha de la Comunitat Valenciana, 
con el fin de recabar información e identificar las zonas sin cobertura en las que intervenir con ayudas públicas.

El objetivo es iniciar un programa de ayudas para extender la cobertura de banda ancha a todos los municipios que carecen de ella, con velocidad de al menos 100 megabits por segundo o superior en núcleos urbanos y 300 megabits por segundo en polígonos industriales.

Además, se ha aprobado la modificación de la Ley de Tasas de la Generalitat para atender una de las demandas del sector de las telecomunicaciones, la eliminación de las tasas al despliegue de fibra óptica en las carreteras. Con ello, se ha facilitado la extensión de la cobertura de banda ancha a las zonas rurales poco pobladas y polígonos industriales, ya que estas tasas ejercían un efecto disuasorio sobre los operadores y paralizaban el despliegue.

Por otra parte, se ha creado una sociedad mercantil del sector público instrumental para la prestación de servicios de comunicaciones electrónicas y de servicios de confianza en las transacciones electrónicas.

Con ella, la Generalitat se va a dotar de una entidad capaz de operar y explotar de manera integral sus centros y redes de telecomunicaciones, fomentando la implantación de redes 5G, y de acometer la renovación tecnológica y la ampliación de la red de difusión del múltiplex autonómico y la radio pública autonómica que utilizará la Corporació Valenciana de Mitjans de Comunicació (CVMC).

En materia de comunicaciones corporativas, la Generalitat se ha decantado por un nuevo contrato centralizado de infraestructuras y servicios para las redes de datos, telefonía fija y móvil e Internet de la Generalitat (CESSTIC). Con él, se ha pasado de una facturación plana a un control exhaustivo del gasto y a una facturación de pago por uso. Esto ha supuesto un ahorro acumulado de 29.5 millones de euros, con una media de ahorro mensual de algo más de 1 millón de euros.

\section{Competencias digitales}

Se ha puesto en marcha el proyecto para la implementación del Marco Europeo de Competencias Digitales (DIGCOMP) en la Comunitat, con el objeto de alinear las acciones de la Generalitat con los trabajos que la Comisión Europea está llevando a cabo en este ámbito. Esta nueva estrategia, alineada con la europea, pretende incrementar las competencias de la ciudadanía y de los empleados públicos mediante acciones de formación.

\section{Educación}

En Educación hemos mejorado la dotación de equipamiento. Se han comprado 70.000 ordenadores para los centros educativos, además de otros dispositivos como 1.700 tabletas digitales, 800 switches, 250 equipos de impresión, entre otros. 
Además, casi la totalidad de los centros de la Comunitat cuentan ya con banda ancha de $100 \mathrm{Mbps}$ y se ha instalado fibra óptica en todos los centros con enseñanzas obligatorias que disponían de cobertura, por lo que más del 70\% de los centros públicos disponen ya de fibra.

Con respecto a la distribución educativa de software libre de la Generalitat, LliureX ha publicado una nueva versión y ha visto renovado tanto su diseño, como su marca y sus funcionalidades, para adaptarse a las necesidades actuales de los centros, las aulas y los hogares.

Por otra parte, los sistemas informáticos que han hecho posible el proyecto Xarxallibres han supuesto un hito importante en la colaboración con las corporaciones locales y las tres diputaciones provinciales.

En el ámbito puramente académico, en septiembre de 2016 se extendió a la totalidad de centros el uso de aplicaciones multidispositivo tanto para el colectivo docente (Módulo Docente 2.0) como las familias (Web Familia 2.0), permitiendo no sólo la consulta de las notas y actividades de los alumnos sino la comunicación bidireccional entre centros, docentes y responsables familiares. El último curso se triplicó su uso, superando ya los 3,5 millones de comunicaciones electrónicas entre las familias y los docentes/secretaría del centro.

Otro hito destacable, ha sido la adjudicación de plazas en los centros educativos dentro del proceso de admisión de alumnado (unas 200.000 solicitudes anuales), al ser realizado por primera vez en concurrencia competitiva mediante algoritmo matemático por el sistema informático ITACA, generando transparencia y eficiencia.

Por último, se ha modernizado todo el proceso selectivo de oposiciones a cuerpos docentes (las más mayoritarias de la historia en 2018 y 2019 con 40.000 solicitudes para 7.630 plazas), gracias a los nuevos sistemas de tramitación electrónica.

\section{Justicia}

Los cambios TIC de esta legislatura en el ámbito de la Administración de Justicia han permitido atender peticiones históricas de las fiscalías y de los profesionales que nunca se habían tenido en cuenta.

Para empezar, se ha conseguido que el expediente judicial electrónico y el proyecto papel cero estén ahora mucho más cerca, ya que se ha incorporado la firma electrónica en todos los procesos de gestión, se ha habilitado la comunicación y presentación telemática de escritos con profesionales y entidades colaboradoras y también el acceso al expediente judicial por la Fiscalía, etc.

Además, se ha conseguido que los profesionales puedan acceder de forma autónoma y segura a las grabaciones de las vistas judiciales, evitando desplazamientos a los juzgados para solicitar copias de los videos.

Para ello, se ha renovado la totalidad del parque informático, adquiriendo 6.500 ordenadores con doble pantalla y más de 700 portátiles que habilitan el trabajo 
remoto de jueces y fiscales. Se ha renovado la totalidad de la red de comunicaciones de banda ancha de los juzgados y se está trabajando en la renovación de las redes locales. Además, en infraestructuras TIC, se han centralizado las 36 bases de datos y servidores locales de los Partidos Judiciales, creando una única base de datos central, lo que ha supuesto una reducción de costes de mantenimiento y de administración técnica.

También se han puesto en marcha otros proyectos como la cita previa en los Registros Civiles, la bolsa única de peritos judiciales y el nuevo sistema de gestión de las Oficinas de Atención a las Víctimas del Delito, que da servicio a una red de 25 oficinas y casi 100 usuarios que atienden a las víctimas.

Como resumen, hay que indicar que las inversiones realizadas en TIC para Justicia superan los 3.700 .000 euros anuales.

\section{Hacienda}

Durante esta legislatura se ha puesto en marcha la Ley de Cuentas Abiertas, por la que se publica la situación de la Tesorería y el detalle de la contabilidad de la Generalitat, Organismos Autónomos y Sector Publico, produciendo un hito para la Comunitat al ser la primera Comunidad Autónoma que publica el detalle de sus cuentas públicas para la consulta de la ciudadanía.

También se ha puesto en marcha la plataforma online de pagos de la Generalitat para que empresas y ciudadanía puedan consultar el estado de todos sus pagos. Además, en materia tributaria, se ha incorporado el pago con tarjeta para ofrecer al contribuyente todas las opciones de pago de impuestos (sea presencial o telemático) y agilizando, así, y mejorando la recaudación tributaria de la Generalitat.

La facturación electrónica ha vivido una gran evolución, pasando de suponer tan solo el 14\% en 2014 al 96,5\% en el año 2019, es decir, prácticamente toda la gestión de la facturación de la Generalitat se realiza ya de manera electrónica, con un volumen medio anual de 750.000 facturas.

Se ha logrado que la contratación pública se realice de manera electrónica en toda la Administración de la Generalitat y su Sector Público, en las primeras fases de la contratación (licitación electrónica), mejorando así la gestión de la contratación de todos los departamentos.

En cuanto a la gestión de nóminas de los funcionarios de diferentes colectivos (administración general, especial, personal docente y personal de justicia), se ha trabajado para agilizar el proceso y disminuir la carga de trabajo en la gestión administrativa de Educación, Justicia y Función Pública.

Así, se procedió a la integración automática con el sistema de liquidación directa de la seguridad social (SILTRA), y durante el año 2018, se ha automatizado, por ejemplo, el $86 \%$ de la gestión anual del personal docente. 
Para finalizar, cabe destacar la elaboración del pliego técnico para la contratación del nuevo sistema económico financiero de la Generalitat (proyecto NEFIS). Esta licitación establece el punto de salida para la implantación de este sistema que permitirá centralizar bajo una misma plataforma la gestión de los procesos de las áreas corporativas económico financieras.

\section{Sistemas de información para otros departamentos}

Cabe destacar el desarrollo de sistemas de información como GVAOberta para la participación ciudadana y la mejora de la transparencia en la Generalitat; la aplicación GVAire para la consulta de la información geolocalizada sobre la calidad del aire en la Comunitat; el registro de incendios forestales para la centralización de datos la trazabilidad de los incendios desde su origen hasta su investigación y estudio; el registro de agentes de cooperación internacional, y la gestión de las solicitudes de ayudas de la Política Agraria Común (PAC), que incluye un módulo de monitorización vía satélite de recintos objeto de ayudas.

En materia de Igualdad y Políticas Inclusivas, en 2019 se trabajó para descentralizar todo el proceso de solicitud de ayudas a la Dependencia hacia las administraciones locales, con el objetivo de mejorar la gestión de los expedientes y la cobertura, lo que ha permitido contabilizar más de 50.000 solicitudes.

También se ha desarrollado el nuevo sistema de información para la gestión integral de los expedientes de la Renta Valenciana de Inclusión, lo que ha permitido registrar más de 30.000 solicitudes; y se ha informatizado la gestión de subvenciones relevantes como los conciertos de la conselleria (servicios sociales, igualdad de género, etc.) y otras ayudas.

Por último, se ha implantado un nuevo modelo informático para la aprobación de los planes urbanísticos, mediante la interacción telemática entre ayuntamientos y Generalitat, con el fin de que la tramitación se realice de manera electrónica para reducir plazos, lo que será un elemento clave para la atracción de inversiones.

\section{La pandemia}

La Generalitat comenzó 2020 con el objetivo de dar continuidad a los planes estratégicos TIC que finalizaron en 2019. Se perseguía culminar la transformación digital de la Administración valenciana con una nueva estrategia encaminada a digitalizar la forma de trabajar en la Generalitat, racionalizar y simplificar la gestión y transformar la cultura organizativa.

Sin embargo, ante la excepcionalidad de los acontecimientos que se vivieron en el mes de marzo, tras la declaración del estado de alarma, se priorizó la labor reactiva y de respuesta ante las necesidades concretas surgidas en el contexto de la crisis sociosanitaria.

Esta labor ha acaparado gran parte de los esfuerzos de los departamentos con competencias en TIC, con el fin de garantizar que la ciudadanía y las empresas 
pudiesen seguir ejerciendo sus derechos y obligaciones a pesar de las restricciones de movilidad impuestas por la pandemia.

\section{Plan para la implantación del teletrabajo y el proyecto FUNCION@gva}

La DGTIC dio inicio al proyecto FUNCION@gva para la transformación digital del personal empleado público. Sin embargo, la iniciativa se encontraba en fases iniciales, por lo que se definió un plan para la implantación del teletrabajo durante los meses de confinamiento.

Este esfuerzo de implantación permitió que una media diaria de 8.000 personas en la Generalitat pudiera desempeñar sus funciones en la modalidad de teletrabajo. Sin embargo, para transformar el modelo productivo del personal de la Generalitat se requieren herramientas TIC que posibiliten la verdadera transformación digital de la Administración.

En ese sentido, el proyecto FUNCION@gva va a transformar la forma de trabajar en la Generalitat, a revolucionar la forma en la que se concibe el puesto de trabajo, incrementando las capacidades digitales de las empleadas y empleados públicos, en el marco de una Administración moderna y más digital.

La puesta en marcha del proyecto comenzó con un piloto para el suministro de licencias software y servicios asociados para facilitar el teletrabajo a 900 personas. El objetivo era generar un nuevo entorno de trabajo, que mejorara el desempeño diario de las funciones del personal, por medio de herramientas informáticas que facilitan la colaboración, la movilidad, el aumento de la productividad y el acceso e intercambio de información desde cualquier dispositivo y ubicación.

Lo que se persigue con FUNCION@gva es mejorar la calidad de los servicios públicos a la ciudadanía, ya que estas herramientas no son exclusivas para el teletrabajo, mejoran el desempeño laboral, en general, pero resultan imprescindibles para ejercer con mayor comodidad y eficacia el trabajo remoto.

\section{Tendencias tecnológicas}

La situación de crisis derivada de la pandemia por la COVID-19 ha supuesto para la Administración Pública un impulso acelerado en la implantación de las tendencias tecnológicas que estaban dando sus primeros pasos: el trabajo remoto o teletrabajo y el uso de soluciones en la nube.

Implantar estas nuevas tendencias de forma masiva ha tenido un gran impacto en la prestación de servicios TIC, las soluciones tecnológicas, los servicios a los usuarios y sobre todo en las estrategias de ciberseguridad.

Para poder dar respuesta a estas necesidades, la Generalitat ha implantado diversas soluciones y ha definido una nueva estrategia de defensa, basada en los nuevos riesgos, para adaptar los sistemas de monitorización y detección y definir soluciones seguras para el trabajo remoto. 


\section{Puestos de trabajo securizados}

La Generalitat ha puesto a disposición de todos sus empleados y empleadas las herramientas básicas necesarias para el teletrabajo, como el acceso remoto al correo electrónico corporativo, y ha ido desplegando los puestos de trabajo securizados para asegurar las tareas críticas y el acceso a las herramientas corporativas más específicas desde los hogares.

Los puestos de trabajo securizados permiten garantizar la seguridad de la información que circula entre los domicilios y la Generalitat y asegurar la confidencialidad de la información que maneja la Administración autonómica y, por tanto, salvaguardar los derechos de la ciudadanía.

Para ello, se amplió la capacidad del sistema de conexión mediante VPN (Virtual Private Network), es decir a la red privada virtual de la Generalitat, para permitir a determinados usuarios y usuarias acceder a una red sin necesidad de estar físicamente conectados y trabajar desde cualquier otro sitio distinto a su oficina.

\section{Certificados digitales y proyecto de videoidentificación}

En cuanto a la certificación que permite verificar la identidad de los usuarios en el mundo virtual, se han realizado las tareas necesarias para facilitar el uso y la obtención de certificados digitales, también de certificados de personal empleado público, y para extender la validez de los ya existentes, que hasta ese momento estaba limitada a cinco años.

En este sentido, la Generalitat desarrolló durante el estado de alarma un nuevo servicio de identificación por videoconferencia para permitir la emisión de certificados electrónicos cualificados, a aquellas personas usuarias que los requiriesen, sin tener que abandonar su hogar y garantizando la seguridad su técnica y sanitaria.

\section{Distribución de equipamiento}

En cuanto a la distribución de equipamiento, se han distribuido cerca de 5.000 ordenadores portátiles entre el personal sanitario, los centros docentes, personal de Justicia y del resto de la Administración de la Generalitat, así como 29.000 tabletas digitales con un bono de datos para alumnado sin recursos, 800 dispositivos para facilitar la comunicación de los pacientes con sus familias en hospitales y residencias, 1.700 enrutadores y cerca de 2.000 tarjetas SIM para familias en riesgo de exclusión y para estudiantes universitarios y 100 ordenadores de sobremesa para los centros de acogida.

\section{Infraestructuras e información}

En cuanto a las infraestructuras TIC, se hizo un esfuerzo para poner en marcha los hospitales de campaña, las nuevas ubicaciones de asistencia sanitaria y los hoteles 
medicalizados, además de nuevos centros de acogida a la mujer, centros de acogida de menores y las residencias de igualdad para la acogida de pacientes no graves, entre otros.

Además, se ha dado especial soporte en la gestión económica de los departamentos correspondientes a Sanidad, Hacienda e Intervención para facilitar la gestión de suministros de material sanitario y se ha adaptado la aplicación corporativa gvContratos para poder incluir las contrataciones de emergencia. Asimismo, se han habilitado nuevos permisos de acceso a sistemas corporativos para facilitar la labor desde los Institutos de Medicina Legal o los juzgados.

Por otra parte, la Generalitat ha desarrollado diversas iniciativas para of recer información actualizada sobre la COVID-19 a la ciudadanía, tanto desde el portal corporativo de la Generalitat como mediante la publicación de nuevos portales y apps, como App GVA Coronavirus o App GVA Responde. Además, se han creado espacios de trabajo colaborativo para el intercambio de información y documentación para atender cuestiones específicas como los ERTE.

Asimismo, se han puesto en funcionamiento cuatro nuevas líneas 900 para responder a las preguntas de la ciudadanía en torno a las crisis del coronavirus y nuevas estaciones base de la red de Comunicaciones Móviles Digitales de Emergencias y Seguridad de la Generalitat (COMDES) para mejorar la cobertura de la red en algunas zonas concretas.

\section{Tramitación electrónica}

La situación generada ha hecho de 2020 un hito en el camino hacia la transformación digital y, más concretamente, la generalización del uso de la administración electrónica entre la ciudadanía. Las cifras así lo indican, ya que respecto al año anterior las tramitaciones electrónicas han crecido un $65 \%$, los accesos a la carpeta ciudadana un $131 \%$, las notificaciones electrónicas un 123\% y un $21 \%$ los pagos on-line.

Además, ante el cierre de oficinas para trámites presenciales, la Generalitat habilitó la tramitación telemática de determinados servicios como la solicitud de registro como demandante de empleo, la prórroga de títulos y carnets de familia numerosa y monoparental, las ayudas directas a los servicios sociales de infancia, etc. También se ha trabajado en un sistema para recabar información sobre la afectación de la pandemia en las residencias de mayores y entre niños y adolescentes.

En 2020, se contabilizaron 2,36 millones de tramitaciones en la plataforma de tramitación de la Generalitat, repartidas entre más de 2.000 trámites electrónicos diferentes. Entre los más tramitados se encuentran los referentes a las ayudas al alquiler de viviendas; los ERTE por causa mayor relacionados con la COVID-19; las ayudas extraordinarias a personas trabajadoras autónomas; o el procedimiento extraordinario para la cobertura de las necesidades de recursos humanos derivados de la pandemia en instituciones sanitarias. 


\section{Formación on-line}

La Generalitat ha multiplicado la actividad de la plataforma de aprendizaje virtual eFormación, debido al aumento de la demanda de formación on-line provocada por el confinamiento y la crisis sanitaria.

La plataforma ofrece aprendizaje electrónico y da servicio a más de 30 organizaciones (Labora, IVAP, IVAJ, CdT, etc.), que la utilizan para ofrecer cursos por Internet desde sus distintos campus. eFormación ofrece herramientas técnicas, de asesoramiento y soporte en eLearning a las organizaciones adheridas, para que puedan ofrecer formación presencial, semipresencial y on-line.

La plataforma da servicio tanto a la ciudadanía, a través del portal SAPS, como a los empleados y empleadas públicos. Durante este año, eFormación ha realizado más de 1.400 acciones formativas, todas ellas accesibles, también, desde dispositivos móviles y tabletas.

\section{Ruedas de prensa telemáticas y videoconferencias}

La habilitación de ruedas de prensa en directo por videoconferencia ha sido uno de los hitos alcanzados en los primeros meses de la pandemia.

Con este sistema, la Generalitat ha sido pionera en España al permitir la realización de ruedas de prensa telemáticas, con el fin de evitar la vulneración del ejercicio del periodismo y como respuesta a la petición de los profesionales de la Comunitat Valenciana.

En este sentido, se han llevado a cabo diversas acciones relacionadas, como la ampliación de la capacidad de videoconferencia en el Consell, la creación de plataformas de videoconferencia específicas para Sanidad, Educación y Justicia y el soporte específico para las videoconferencias con el Estado y las reuniones del Consell.

\section{Plan MULAN de digitalización de la educación}

Hasta la declaración del estado de alarma, la enseñanza por medios digitales en la Comunitat se circunscribía a ciclos formativos semipresenciales y al centro específico de educación a distancia (CEED). Las plataformas disponibles y ampliamente extendidas de e-learning, como Moodle, no estaban concebidas para su uso en otros niveles educativos, ya que no estaba previsto implantar modelos de educación a distancia exclusiva.

EI Plan MULAN permitió establecer un marco tecnológico con las herramientas desarrolladas por la Generalitat, permitiendo una digna salida al cierre presencial de todos los centros educativos.

MULAN fue diseñado a partir de las plataformas informáticas disponibles en aquel momento: 
19.1. Extensión de la plataforma AULES: es un entorno virtual de aprendizaje (EVA) desarrollado por la Generalitat sobre Moodle, concebido inicialmente para la FP semipresencial y unos 3.000 alumnos. Para su uso en el resto de etapas, se planificó una entrada escalonada del alumnado y profesorado. Durante los meses de confinamiento, AULES se extendió para llegar a 200.000 alumnos y 40.000 profesores.

19.2. Generalización de la plataforma Web Familia y nueva app: desarrollada para facilitar la relación entre los centros y docentes con las familias y el alumnado, pasó de ser utilizada por unas 250.000 personas, de forma voluntaria, a que se extendiese a la totalidad de los centros para su uso generalizado.

19.3. PORTALEDU: las páginas web de cada uno de los centros educativos multiplicaron sustancialmente el número de visitas, ofreciendo información diaria útil sobre las condiciones propias de cada centro y las instrucciones dictadas por la Generalitat.

19.4. Videoconferencias: las videoconferencias se convirtieron en una herramienta necesaria durante el confinamiento. Gracias al ofrecimiento altruista de las empresas CISCO y Telefónica, se pudo implantar, en pocos días, el uso de Webex para el profesorado y alumnado.

19.5. Equipamiento para inclusión digital del alumnado: la Generalitat fue pionera en la adquisición de tabletas durante el confinamiento. Se adquirieron, inicialmente, 14.000 dispositivos para el alumnado en riesgo de exclusión digital y se repartieron, junto a un bono de datos, individualmente en sus domicilios. Para el inicio del curso 2020-2021, se adquirieron 15.000 tabletas más, que se repartieron en los centros educativos. Para el alumnado que aún resta en riesgo de exclusión, está prevista la adquisición de 15.000 tabletas (infantil y primaria) y 35.000 portátiles (secundaria, bachillerato y ciclos formativos).

19.6. Telematrícula.es: destaca el novedoso proceso para la tramitación online de la admisión escolar para todas las etapas educativas, que se realizó a través del portal Telematricula.es, para solicitar plaza en los centros educativos sostenidos con fondos públicos. El procedimiento fue exclusivamente telemático, gracias a la introducción de un nuevo concepto, la clave de admisión.

\section{Colaboración público-privada en Educación}

20.1. Videoconferencias: gracias al ofrecimiento altruista de las empresas CISCO y Telefónica, se pudo implantar el uso de Webex para el profesorado y alumnado de etapas críticas, para clases en directo, tutorías y tareas digitales. Al perdurar el confinamiento, se amplió a 
otras etapas como infantil, primaria, ESO, escuelas oficiales de idiomas y conservatorios de música.

20.2. Convenio con Microsoft: La Generalitat ha firmado un convenio de colaboración público-privada con Microsoft Ibérica para reforzar la plataforma educativa digital de la Generalitat y la formación a distancia con aplicaciones. Se facilitará el acceso a un entorno de aprendizaje en la nube, a disposición del profesorado y el alumnado de los centros sostenidos con fondos públicos en la Comunitat.

\section{Televistas y agenda de señalamientos judiciales}

21.1. Televistas: La Administración de Justicia de la Comunitat Valenciana dispone de una nueva herramienta para celebrar televistas. Se trata de vistas orales en las que la presencia física de los intervinientes se suple mediante el uso de sistemas de videoconferencia, respetando y asegurando las mismas garantías jurídicas en la grabación del juicio telemático como en su modalidad presencial.

21.2. Agenda de señalamientos judiciales: la Generalitat ha puesto en marcha un nuevo servicio que permite consultar a través de Internet la fecha, hora y lugar de celebración de los señalamientos correspondientes a procedimientos judiciales de los Órganos Judiciales de la Comunitat Valenciana.

\section{GEN DIGITAL 2025}

Se hace necesario establecer un nuevo plan que sirva de marco general en el que ordenar todas las acciones que posibiliten un claro avance en la digitalización de la Generalitat y permitan profundizar en la transformación digital.

Además, esta estrategia deberá sustentar el desarrollo de las distintas acciones que emanen del acuerdo Alcem-nos, con el que la Generalitat y el resto de agentes sociales, políticos y económicos han sentado las bases para la recuperación económica y social de la Comunitat Valenciana tras la emergencia sanitaria.

Para la definición de este plan se han tenido en cuenta las políticas digitales aprobadas por el Gobierno de España y la Comisión Europea, así como los Objetivos de Desarrollo Sostenible (ODS) de Naciones Unidas.

La Agenda España Digital 2025 identifica al menos dos objetivos estratégicos en los que tiene reflejo en GEN Digital 2025, como son los dirigidos a impulsar la digitalización de las Administraciones Públicas y a garantizar los derechos de la ciudadanía en el nuevo entorno digital.

La misión de GEN Digital 2025 es culminar la transformación digital de la Administración del Consell y sus organismos autónomos, digitalizando la forma de 
trabajar, racionalizando y simplificando la gestión y los procesos internos y transformando la cultura organizativa de la Generalitat.

GEN Digital 2025 pretende convertir a la Administración de la Generalitat en una organización pública digital, cohesionada y colaborativa, capaz de trabajar, dar servicio y relacionarse con la sociedad como un ente único y ágil, gracias a una estrategia compartida y abierta, basada en el uso de las TIC como facilitadoras de la transformación y el cambio cultural.

Por otra parte, el 10 de noviembre de 2020, se alcanzó un acuerdo en el Consejo de la UE sobre el próximo presupuesto a largo plazo y la iniciativa Next GenerationEU para impulsar la recuperación. Este acuerdo pretende ayudar a reconstruir la Europa posterior a la COVID-19, que será más digital, además de ecológica y resiliente.

En este sentido, el programa Europa Digital identifica distintas líneas de trabajo para contribuir a la transformación. Entre ellas, destacan las destinadas a reforzar la soberanía digital; mejorar los servicios digitales; establecer una estrategia europea de datos; desarrollar la inteligencia artificial; impulsar el uso de tecnologías facilitadoras; garantizar la conectividad y la ciberseguridad; transformar los sectores sanitario y asistencial; el sector educativo y la formación; y digitalizar la justicia.

Por tanto, y considerando la coyuntura actual, GEN Digital 2025 recoge un conjunto de acciones y proyectos, articulados en una estructura de 6 ejes estratégicos:

1. Infraestructuras, comunicaciones y movilidad imprescindibles y críticas

2. Gobierno inteligente

3. Gobierno sostenible

4. Educación digital

5. Sanidad y servicios sociales eficientes

6. Justicia moderna

El primer eje estratégico, 'Infraestructuras, comunicaciones y movilidad imprescindibles y críticas', persigue dotar a la Generalitat de las infraestructuras avanzadas que sus sistemas de información necesitan.

GEN Digital 2025 contiene ciertos proyectos bandera que serán verdaderos impulsores de la transformación digital de la Generalitat. En el caso de este primer eje se han destacado dos, FUNCION@gva y Cloud First.

La iniciativa FUNCION@gva persigue la transformación del modelo de desempeño laboral del personal empleado público de la Generalitat. Cloud First es el proyecto de la Generalitat que suma tecnología y sostenibilidad con el uso de infraestructura en la nube.

El segundo eje, 'Gobierno inteligente' persigue mejorar la atención directa a la sociedad, acercando la Administración a las personas físicas y jurídicas, mediante sistemas de fácil utilización, reduciendo la brecha digital existente para los colectivos con más limitaciones de acceso a la tecnología. 
También se han destacado dos proyectos estrella en este eje: Ànima y NEFIS. Ànima es una iniciativa que quiere poner en valor los datos, habilitando a la Generalitat para explotar de forma intensiva la información y el conocimiento y construyendo una Administración Pública más eficaz y eficiente. NEFIS es un proyecto modernizador que servirá como instrumento indispensable para el seguimiento más efectivo del cumplimiento de los objetivos de estabilidad presupuestaria.

En lo que se refiere al eje 'Gobierno Sostenible', la Generalitat ha hecho una apuesta firme por la sostenibilidad y las tecnologías de la información y la comunicación son importantes aliadas en este propósito.

Este plan contribuye a alcanzar los Objetivos de Desarrollo Sostenible proporcionando infraestructuras fiables, sostenibles, resilientes y de calidad, y una indirecta, facilitando la implementación de las políticas de la Generalitat que permiten ofrecer servicios de calidad en atención sanitaria, inclusión, igualdad, educación, turismo, cambio climático y demás iniciativas de gobierno claramente relacionadas con la consecución de estos objetivos.

El proyecto PICA será la base del Internet de la Cosas aplicado al territorio. Este sistema facilitará la vigilancia activa que permita avanzar en el control de los indicadores medioambientales definidos para la prevención, monitorización y control integral de la contaminación, garantizará la implantación del dato único como principio de actuación en todos los procesos y permitirá avanzar en el gobierno abierto, pudiendo ofrecer una mejor transparencia mediante la apertura de datos de forma consistente, unificada e integral.

El cuarto eje estratégico 'Educación Digital' persigue vertebrar un conjunto de acciones que posibiliten la transformación del modelo educativo valenciano, dotando a la comunidad de las herramientas tecnológicas necesarias para la adaptación de las personas a este cambio de paradigma digital.

El proyecto Centre a Casa facilitará la relación entre todos los agentes implicados en el proceso, mediante el uso de nuevas plataformas para la colaboración, la comunicación y la tramitación electrónica.

En este eje se ha destacado el proyecto Centro Digital Colaborativo, que es el nuevo modelo innovador de funcionamiento de los centros educativos de la Comunitat Valenciana, con el que se pretende facilitar la relación entre los equipos directivos de los centros, los docentes, las familias y el alumnado, mediante el uso de nuevas plataformas para la colaboración, la comunicación y la tramitación electrónica y que van a posibilitar una auténtica transformación digital de toda comunidad.

En el ámbito de los servicios sociales, las líneas estratégicas persiguen proporcionar una atención más ágil a la ciudadanía, mediante la remodelación tecnológica de los sistemas de información actuales. En virtud del establecimiento del contrato programa para la financiación de la red de servicios sociales de la Comunitat Valenciana, se materializará un convenio de colaboración con las entidades locales para facilitar la asistencia social en todos los municipios y la Renta de Inclusión dotará a la ciudadanía del ingreso mínimo para una vida digna. 
Por otra parte, en materia de integración sociosanitaria, la línea estratégica describe la construcción del nuevo Espacio Sociosanitario, que permitirá mantener una visión unificada de las necesidades de la población en ambas facetas, la sanitaria y la social, mediante el establecimiento de la historia sociosanitaria de cada ciudadano y ciudadana, que permita abordar, desde una visión holística, el cuidado de la salud y la asistencia social requerida, integrando a todos los agentes involucrados.

En cuanto al ámbito sanitario, las líneas estratégicas persiguen consolidar una sanidad cercana, robusta, integrada y prospectiva. la promoción de la salud y la asistencia sanitaria logran sus mayores cotas de eficiencia y efectividad cuando el sistema sanitario consigue hacer partícipe a la ciudadanía de las acciones que se llevan a cabo.

Por todo ello, el Espacio Sociosanitario es proyecto insignia del eje 'Sanidad y Servicios Sociales eficientes'. Es el área de convergencia de los servicios sociales y sanitarios que permitirá mantener una visión unificada de las necesidades de la población en ambas facetas. Mediante el establecimiento de la historia sociosanitaria de cada ciudadano y ciudadana, se podrá abordar, desde una visión holística, el cuidado de la salud y la asistencia social requerida, integrando a todos los agentes involucrados y prestando la atención especializada más adecuada en cada caso.

Y finalmente, el eje 'Justicia moderna' implementa la estrategia en la Administración de Justicia de la Generalitat, alineada con la búsqueda de una justicia moderna para nuestra sociedad, una justicia que sea cercana y dónde la ciudadanía sea la protagonista del procedimiento y tenga a su disposición mecanismos ágiles para la solución de conflictos, todo cimentado sobre los valores de eficacia y transparencia. Just@cv es el proyecto destacado de este eje. Pretende facilitar el acceso de la ciudadanía y los profesionales y operadores jurídicos a la Administración de Justicia, a través de los servicios de la Sede Judicial Electrónica, y prestar un servicio eficiente, mediante el aprovechamiento de las ventajas que ofrece el uso de la tecnología. 